\title{
Effects of combined olmesartan and pravastatin on glucose intolerance and cardiovascular remodeling in a metabolic-syndrome model
}

\author{
Mizuki Mizukawa $^{1}$, Koji Ohmori ${ }^{1}$, Ayumi Obayashi ${ }^{1}$, Yasuhiro Ishihara ${ }^{1}$, Junji Yoshida ${ }^{1}$, Takahisa Noma ${ }^{1}$, \\ Kazushi Yukiiri ${ }^{1}$, Hiroaki Kosaka ${ }^{2}$ and Masakazu Kohno ${ }^{1}$
}

Hypertension and dyslipidemia frequently coexist in patients with progressive insulin resistance and thus constitute metabolic syndrome. We sought to determine the merits of combining an angiotensin II receptor blocker and a 3-hydroxy-3-methylglutarylcoenzyme A reductase inhibitor in treating this pathological condition. Five-week-old Otsuka Long-Evans Tokushima Fatty rats, a model of metabolic syndrome, were untreated or treated with olmesartan $3 \mathrm{mg} \mathrm{kg}^{-1}$ per day, pravastatin $30 \mathrm{mg} \mathrm{kg}^{-1}$ per day or their combination for 25 weeks. Long-Evans Tokushima Otsuka rats served as normal controls. The antihypertensive effect of olmesartan and the lipid-lowering properties of pravastatin were both augmented by the combination. The oral glucose tolerance test revealed that only the combined treatment significantly reduced the area under the time-glucose curve, which was accompanied by augmented adiponectin messenger RNA expression in epididymal adipose tissue. Although the total cardiac endothelial nitric oxide synthetase (eNOS) content did not significantly differ among the groups, the combined treatment significantly increased the content of dihydrofolate reductase, a key eNOS coupler. Dihydroethidium staining of the aorta showed that the combination most significantly attenuated superoxide production. Moreover, Azan-Mallory staining revealed that the combination most significantly limited the perivascular fibrosis and wall thickening of intramyocardial coronary arteries. In conclusion, the combination of olmesartan and pravastatin augmented adiponectin expression in white adipose tissue and improved glucose tolerance in a rat model of metabolic syndrome, which was associated with more significant ameliorations of cardiovascular redox state and remodeling than those by treatments with either agent alone.

Hypertension Research (2009) 32, 617-624; doi:10.1038/hr.2009.63; published online 22 May 2009

Keywords: adiponectin; diabetes mellitus; dihydrofolate reductase; endothelial nitric oxide synthetase; oxidative stress

\section{INTRODUCTION}

Several clinical studies have shown that inhibition of the reninangiotensin system substantially lowers the risk of developing type II diabetes mellitus $(\mathrm{DM})^{1,2}$ and improves insulin sensitivity. ${ }^{3}$ Olmesartan (OLM), an angiotensin II type-1 receptor (AT1) blocker (ARB), was reported to reduce oxidative stress in adipose tissue and to ameliorate adipocytokine dysregulation in obesity models, which was associated with increased levels of plasma adiponectin and improved insulin sensitivity. ${ }^{4}$ Pravastatin (PRA), a 3-hydroxy3-methylglutaryl-coenzyme A reductase inhibitor (statin), also prevents new onset of $\mathrm{DM}^{5}$ and improves glucose tolerance in patients. ${ }^{6-8}$ We and others have shown that PRA upregulates adiponectin messenger RNA expression in adipose tissue ${ }^{8}$ and in cultured adipocytes, ${ }^{9}$ and prevents the development of DM in animal models. ${ }^{9-11}$ Clinical studies have also shown that PRA treatment increases plasma adipo- nectin in patients with hypercholesterolemia and is associated with improved glucose metabolism. ${ }^{8,9}$ As dyslipidemia often accompanies hypertension, such individuals are frequently treated with both reninangiotensin system inhibitors and statins. Several preclinical studies have shown that ARBs and statins additively or synergistically prevent cardiovascular remodeling. ${ }^{12,13}$ However, the effect of the combined therapy on glucose intolerance, which often coexists with other risks and worsens prognosis by increasing cardiovascular oxidative stress, remains to be investigated.

5,6,7,8-tetrahydrobiopterin (BH4), an essential cofactor for endothelial nitric oxide synthetase (eNOS), tends to be oxidized to 7,8 -dihydrobiopterin $(\mathrm{BH} 2)$ in DM. ${ }^{14}$ Decreased BH4 limits eNOS dimerization, resulting in decreased nitric oxide $(\mathrm{NO})$ synthesis and increased superoxide production, so-called eNOS uncoupling, and is a major mechanism of endothelial dysfunction. ${ }^{14}$ In this regard,

${ }^{1}$ Department of Cardiorenal Cerebrovascular Medicine, Kagawa University Faculty of Medicine, Kagawa, Japan and ${ }^{2}$ Department of Cardiovascular Physiology, Kagawa University Faculty of Medicine, Kagawa, Japan

Correspondence: Dr K Ohmori, Department of Cardiorenal Cerebrovascular Medicine, Kagawa University Faculty of Medicine, 1750-1 Ikenobe, Miki-cho, Kagawa 761-0793, Japan.

E-mail: komori@med.kagawa-u.ac.jp

Received 23 February 2009; revised 31 March 2009; accepted 8 April 2009; published online 22 May 2009 
dihydrofolate reductase (DHFR) plays an important role in eNOS coupling by recycling $\mathrm{BH} 2$ to preserve $\mathrm{BH} 4 .{ }^{15}$ However, especially under accentuated oxidative stress such as that caused by angiotensin II stimulation ${ }^{15}$ or DM, ${ }^{16}$ DHFR expression is reduced and $\mathrm{BH} 2$ is not recycled, which causes eNOS uncoupling and further aggravates oxidative stress.

In the present study, we investigated whether the combination of OLM and PRA would synergistically affect glucose intolerance in a model of metabolic syndrome. We also examined the effects of the combined therapy on adiponectin expression in white adipose tissue (WAT), and on the cardiovascular redox state and the resultant remodeling.

\section{METHODS}

\section{Animals and experimental protocol}

The experiments were conducted according to the National Research Council's guidelines. Male Otsuka Long-Evans Tokushima Fatty (OLETF) rats fed with normal chow were treated with either OLM $\left(3 \mathrm{mg} \mathrm{kg}^{-1}\right.$ per day), PRA ( $30 \mathrm{mg} \mathrm{kg}^{-1}$ per day: $30 \%$ of the full dose tested in our earlier studies ${ }^{10,11}$ ), or their combination (OLM+PRA) ( $n=12$ for each) from 5 weeks of age. Agents were suspended in drinking water at concentrations adjusted according to the amount of water consumed during the preceding 2 days, so that the total scheduled doses were administered. The untreated controls were OLETF rats given standard drinking water $(n=12)$, while the Long-Evans Tokushima Otsuka (LETO) rats, which do not develop DM, served as normal controls $(n=12)$. All animals were housed in pairs at a pathogen-free facility under controlled temperature $\left(23^{\circ} \mathrm{C}\right)$ and humidity $(55 \%)$, with a 12-h artificial lightdark cycle at the Division of Animal Experiment, Life Science Research Center in Kagawa University. The animals and agents were provided by Otsuka Pharmaceutical Co. Ltd., Tokyo, Japan and by Daiichi-Sankyo Co. Ltd., Tokyo, Japan, respectively.

Blood pressure (BP) was measured by the tail-cuff method at 10, 20 and 30 weeks of age. At 30 weeks of age, blood was sampled from the right jugular vein after an overnight fast and the oral glucose $\left(2 \mathrm{~g} \mathrm{~kg}^{-1}\right.$ body weight $)$ tolerance test (OGTT) was applied as described elsewhere. ${ }^{10,11,17}$ Then, all the rats were killed after administering deep anesthesia with i.p. sodium pentobarbital (50 $\mathrm{mg} \mathrm{kg}^{-1}$ ), and the heart, aorta and epididymal WAT were excised.

\section{Biochemical analyses of blood samples}

Blood glucose, total cholesterol, triglycerides and free fatty acid were measured. Plasma insulin, leptin and adiponectin were measured using enzyme-linked immuno-sorbent assay (ELISA) kits (Morinaga Institute of Biological Science Inc., Yokohama, Japan; leptin and adiponectin: Otsuka Pharmaceutical Co. Ltd., Tokyo, Japan). Insulin resistance was evaluated based on the product of fasting glucose and insulin concentrations..$^{10,11}$

\section{Tissue processing}

Short-axis sections (3-mm thick) of the left ventricle at the mid-ventricular level and aorta samples were fixed with formalin or frozen in an optimal cutting temperature (OCT) compound and stored at $-80^{\circ} \mathrm{C}$ until histological assessment. The remaining tissue samples and WAT were immediately frozen in liquid nitrogen and stored at $-80^{\circ} \mathrm{C}$ before total RNA and/or protein extraction.

\section{Histochemical and histological analysis}

Superoxide production in the aorta was detected by dihydroethidium staining, as described earlier. ${ }^{18}$ In brief, tissue segments frozen in OCT compound were sectioned in 3- $\mu \mathrm{m}$-thick slices and then incubated in a light-protected chamber at $37^{\circ} \mathrm{C}$ for $30 \mathrm{~min}$ with $2 \mu \mathrm{M}$ dihydroethidium. ${ }^{18}$ Images were obtained using an Olympus LSM-GB 200 laser scanning confocal microscope equipped with a krypton/argon laser (Olympus Corporation, Tokyo, Japan), using identical acquisition settings. Dihydroethidium fluorescence intensity was quantified using Image J (National Institutes of Health, Bethesda, MD, USA), with regions of interest covering the entire section of the aorta.

The wall thickness of intramyocardial coronary arteries and degree of their perivascular fibrosis were assessed by light microscopy at $\times 400$ magnification using Azan-Mallory stain. We determined the wall-to-lumen ratio (medial thickness compared with luminal diameter) and the ratio of the area of perivascular fibrosis to the vascular cross-sectional area in 5-10 cross-sections of arteries between 30 and $200 \mu \mathrm{m}$ in diameter, as described earlier, ${ }^{10,11,19}$ which were reported as percentage of the values for the normal LETO rats.

\section{Assessment of adiponectin messenger RNA expression in white adipose tissue}

cDNA was synthesized from $500 \mathrm{ng}$ of the total RNA extracted from $400 \mathrm{mg}$ of epididymal WAT using a First-Strand cDNA Synthesis Kit (Roche Diagnostics KK, Tokyo, Japan). We measured the expression of adiponectin ${ }^{10}$ and $18 \mathrm{~S}$ ribosomal RNA (internal control) using real-time reverse transcription polymerase chain reaction (RT-PCR) and an ABI Prism 7000 system (Applied Biosystems Japan Ltd., Tokyo, Japan). We used a TaqMan Rodent 18 S Control Reagents VIC Probe Kit for $18 \mathrm{~S}$ and Assay-on-Demand Gene Expression Products Kits for adiponectin (both from Applied Biosystems Japan Ltd.), with the primer sequences and protocols provided by the manufacturer.

\section{Western blots of eNOS and DHFR}

We measured eNOS ${ }^{10,11}$ and DHFR levels in the heart. The left ventricular tissue was homogenized in lysis buffer and then the protein concentration was determined using a Bio-Rad Protein Assay Kit (Bio-Rad Laboratories KK, Tokyo, Japan). Protein $(20 \mu \mathrm{g})$ was separated and electro-blotted onto nitrocellulose membranes (Bio-Rad Laboratories KK). Blots were incubated with the respective primary monoclonal antibodies for eNOS (1:500) (Sigma-Aldrich Japan KK, Tokyo, Japan) or DHFR (1:2500) (BD Biosciences, San Jose, CA, USA) in phosphate-buffered saline-Tween containing $5 \%$ fat-free milk, probed with anti-mouse horseradish peroxide (HRP)-linked secondary antibody (1:3000) and detected using the ECL plus Western Blotting Detection System (GE Healthcare UK Ltd., Buckinghamshire, UK).

\section{Statistical analysis}

Data are expressed as means \pm s.e.m. Differences in mean values among the groups at one time point and over the time course were analyzed by one-way and two-way analysis of variance (ANOVA), respectively, followed by all pairwise comparisons using the Student-Newman-Keuls test. A level of $P<0.05$ was considered statistically significant.

\section{RESULTS}

\section{Body weight and food intake}

The OLETF rats exhibited obesity, increased epididymal WAT weight and food intake compared with the normal LETO rats. Although the inhibition of obesity did not reach a significant level by either drug or by the combination as compared with the untreated OLETF rats, the increase in epididymal WAT weight, representing visceral adipose tissue weight, was significantly limited by OLM and was further enhanced by adding PRA. None of the therapies affected the amount of food intake (Table 1).

\section{Effects on blood pressure and plasma lipid levels}

Mean BP and lipid levels progressively elevated in the untreated OLETF rats, when compared with the control-LETO rats. The BP increase was significantly limited by OLM, which was enhanced by adding PRA at 30 weeks of age (Figure 1). PRA prevented lipid elevation as expected. OLM also ameliorated dyslipidemia, which was augmented by combining both agents (Table 1). Thus, co-administration further enhanced the primary pharmacological actions of OLM and PRA.

\section{Glucose metabolism and adipokines}

Both fasting blood glucose and insulin levels, and therefore their product, the insulin resistance index, were higher in all OLETF rat groups than in the LETO rats at 30 weeks of age. However, the progression of insulin resistance was significantly limited in groups 
Table 1 Comparisons of body weight, adipose tissue weight, hemodynamics, lipids, glucose metabolism and adipocytekines among treatment groups at 30 weeks of age

\begin{tabular}{|c|c|c|c|c|c|}
\hline & Untreated & PRA & OLM & $O L M+P R A$ & LETO \\
\hline Body weight (g) & $675.8 \pm 24.8$ & $689.0 \pm 11.6$ & $674.3 \pm 14.7$ & $623.9 \pm 13.1^{\dagger}$ & $496.9 \pm 7.8^{*, \dagger, \ddagger, \S}$ \\
\hline WAT weight (g) & $19.3 \pm 1.3$ & $18.1 \pm 0.7$ & $15.8 \pm 1.2^{*}$ & $13.1 \pm 0.5^{*, \dagger}$ & $9.2 \pm 0.4^{*, \dagger, \ddagger, \S}$ \\
\hline Food intake (g per day) & $36.0 \pm 1.0$ & $30.6 \pm 2.1$ & $31.2 \pm 1.3$ & $36.9 \pm 2.8$ & $18.9 \pm 0.6^{*, \dagger, \ddagger, \S}$ \\
\hline Mean BP $(\mathrm{mm} \mathrm{Hg})$ & $132.3 \pm 2.7$ & $126.0 \pm 2.0$ & $120.0 \pm 2.6^{*}$ & $112.5 \pm 2.1^{*, \dagger, \frac{\ddagger}{\dagger}}$ & $105.3 \pm 1.17^{*, \dagger, \ddagger, \S}$ \\
\hline Heart rate $\left(\mathrm{min}^{-1}\right)$ & $498 \pm 7$ & $500 \pm 6$ & $490 \pm 5$ & $478 \pm 5$ & $444 \pm 7^{*, \uparrow, \ddagger, \S}$ \\
\hline Total cholesterol (mg $100 \mathrm{ml}^{-1}$ ) & $170.8 \pm 7.7$ & $130.0 \pm 10.7^{*}$ & $102.2 \pm 5.5^{*, \dagger}$ & $94.7 \pm 6.9 *$, & $88.0 \pm 1.7^{*, \dagger}$ \\
\hline Triglyceride (mg $100 \mathrm{ml}^{-1}$ ) & $388.8 \pm 17.1$ & $248.7 \pm 94.8^{*}$ & $183.5 \pm 24.2^{*}$ & $90.3 \pm 21.1^{*}$ & $36.5 \pm 4.4^{*, \dagger}$ \\
\hline Free fatty acid $\left(\mathrm{mEq} \mathrm{I}^{-1}\right)$ & $510.8 \pm 28.1$ & $608.5 \pm 28.0$ & $687.5 \pm 76.3$ & $437.7 \pm 43.5$ & $415.3 \pm 23.0$ \\
\hline Fasting glucose (mg $100 \mathrm{ml}^{-1}$ ) & $171.0 \pm 10.6$ & $148.4 \pm 4.5^{*}$ & $137.1 \pm 6.4^{*}$ & $131.4 \pm 3.9 *$ & $117.6 \pm 3.4^{*, \dagger}$ \\
\hline Fasting insulin (ng $100 \mathrm{ml}-1$ ) & $1.68 \pm 0.28$ & $1.35 \pm 0.17$ & $0.83 \pm 0.08^{*}$ & $0.95 \pm 0.13^{*}$ & $0.74 \pm 0.07 *$ \\
\hline IR index (mg $\left.100 \mathrm{ml}^{-1} \times \mathrm{ng} 100 \mathrm{ml}^{-1}\right)$ & $238.4 \pm 42.6$ & $164.6 \pm 27.1^{*}$ & $105.9 \pm 13.2^{*}$ & $110.8 \pm 19.7^{*}$ & $89.1 \pm 5.6^{*}$ \\
\hline Leptin $\left(\mu g \mathrm{ml}^{-1}\right)$ & $5.97 \pm 0.73$ & $5.83 \pm 0.64$ & $5.14 \pm 0.34$ & $3.76 \pm 0.43^{* \dagger}$ & $1.56 \pm 0.30 *, \dagger, \ddagger, \S$ \\
\hline Adiponectin $\left(\mu \mathrm{g} \mathrm{ml}^{-1}\right)$ & $2.32 \pm 0.24$ & $2.69 \pm 0.24$ & $3.00 \pm 0.26$ & $3.65 \pm 0.50 *$ & $2.19 \pm 0.11^{\S}$ \\
\hline
\end{tabular}

Abbreviations: BP, blood pressure; IR, insulin resistance; LETO, normal control Long-Evans Tokushima Otsuka rats; untreated OLETF, Otsuka Long-Evans Tokushima Fatty rats; OLM, OLETF rats treated with olmesartan; PRA, OLETF rats treated with pravastatin; OLM+PRA, OLETF rats treated with both pravastatin and olmesartan; WAT, white adipose tissue.

Values are means \pm s.e.m.

${ }^{\star} P<0.05$ vs. Untreated; ${ }^{\dagger} P<0.05$ vs. PRA; ${ }^{\ddagger} P<0.05$ vs. OLM; ${ }^{\circledR} P<0.05$ vs. OLM+PRA by one-way ANOVA and Student-Newman-Keuls test.

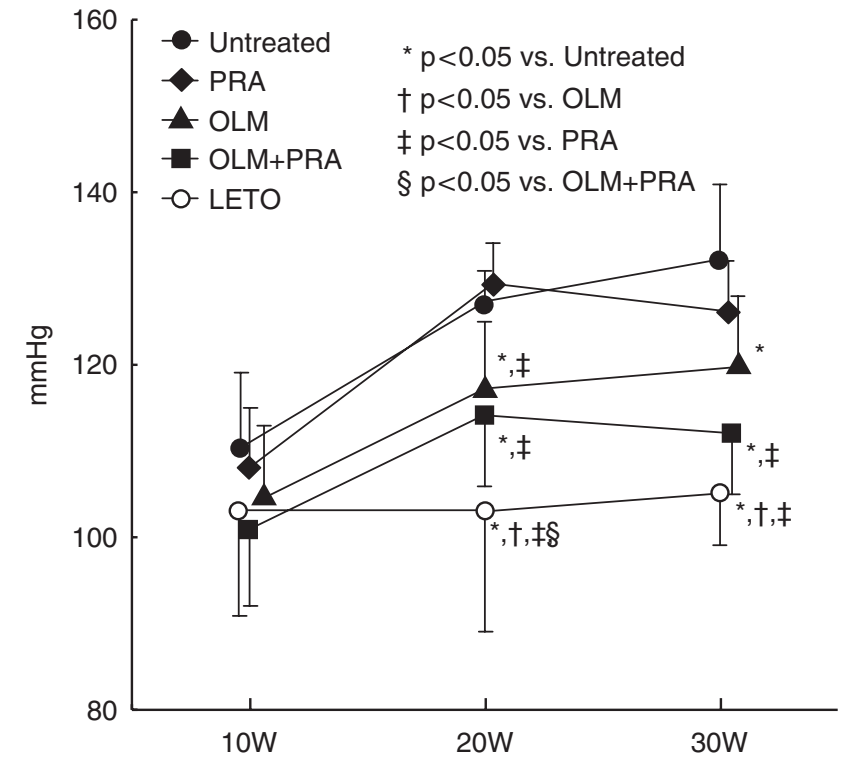

Figure 1 Time course of mean blood pressure. Untreated, untreated Otsuka Long-Evans Tokushima Fatty (OLETF) rats; pravastatin (PRA), OLETF rats treated with PRA (30 $\mathrm{mg} \mathrm{kg}^{-1}$ per day); olmesartan (OLM), OLETF rats treated with olmesartan ( $3 \mathrm{mg} \mathrm{kg}^{-1}$ per day); OLM+PRA, OLETF rats given pravastatin $(30 \mathrm{mg} / \mathrm{kg} /$ day $)$ plus OLM $\left(3 \mathrm{mg} \mathrm{kg}^{-1}\right.$ per day); Long-Evans Tokushima Otsuka (LETO), LETO rats as normal controls. $n=8-11$ for each group. $P$-values are for two-way analysis of variance (ANOVA) and StudentNewman-Keuls test.

given either agent alone or in combination (Table 1). The plasma level of leptin was reduced and that of adiponectin was increased in the OLM+PRA group compared with the untreated OLETF rats (Table 1).

\section{Glucose tolerance}

Figure 2a shows glucose levels during the OGTT at 30 weeks of age. In both monotherapy groups, the glucose levels at 30 and 60 min were significantly decreased compared with the untreated controls (all $P<0.05$ ), but remained elevated at $120 \mathrm{~min}$ (both non-significant $v s$. untreated OLETF rats). However, the combination of OLM and PRA further decreased the blood glucose within 30-120 min $(P<0.05$ vs. PRA at $30 \mathrm{~min}, P<0.05$ vs. both PRA and OLM at 60 and $120 \mathrm{~min}$ ). Thus, the reduction in the area under the time-glucose curve during the OGTT reached a significant level only in the OLM+PRA group (Figure 2b).

\section{Adiponectin messenger RNA expression in WAT}

Adiponectin messenger RNA expression in WAT of the untreated OLETF rats was reduced to $41 \%$ of that in the normal LETO rats. Although neither PRA nor OLM alone significantly upregulated adiponectin messenger RNA expression, OLM+PRA significantly augmented the expression compared with the untreated OLETF rats $(P<0.05)$, to the level of the normal LETO rats (Figure 3a).

\section{Plasma adiponectin levels normalized to adipose tissue weight}

As plasma adiponectin levels are determined not only by the function of adipose tissue but also by adipose tissue mass, ${ }^{20}$ according to earlier reports on the pre-advanced stage of diet-induced obesity models, ${ }^{21,22}$ plasma adiponectin levels were normalized by the weight of epididymal WAT. ${ }^{22}$ The plasma adiponectin/WAT weight ratio was significantly decreased in the untreated OLETF rats down to $48 \%$ of the normal value LETO rats $(P<0.05)$. Olmesartan alone almost completely $(90 \%$ of LETO rats) prevented the decrease $(P<0.01$ vs. the untreated OLETF rats). The combination of OLM and PRA further significantly preserved the ratio ( $118 \%$ of the LETO rats, $P<0.001 \mathrm{vs.}$ the untreated OLETF rats) (Figure $3 \mathrm{~b}$ ).

\section{Aortic superoxide production}

Dihydroethidium staining of the aorta revealed obviously increased superoxide production in the untreated OLETF rats, and that both PRA alone and OLM alone reduced superoxide production (both $P<0.01$ vs. untreated OLETF rats). However, OLM+PRA further significantly reduced superoxide production in the aorta $(P<0.001$ vs. untreated OLETF rats) to a level similar to that of the LETO rats (Figure 4).

\section{Cardiac eNOS and DHFR content}

The eNOS protein content did not significantly differ among the groups (Figure 5a). In contrast, the DHFR content was decreased in the untreated OLETF rats, which was sub-significantly recovered by 

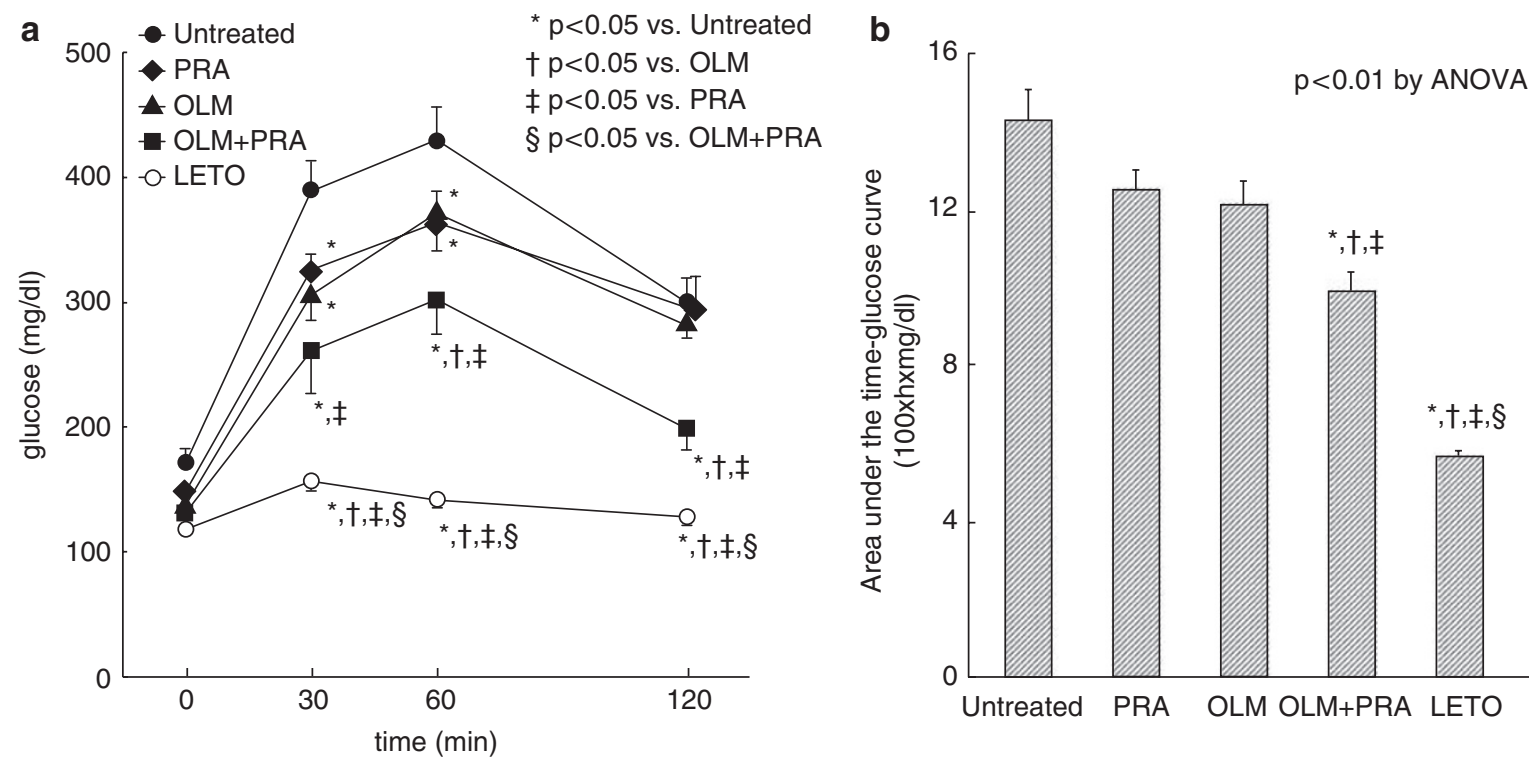

Figure 2 (a) Time course of blood glucose level during the oral glucose tolerance test ( $2 \mathrm{~g} \mathrm{~kg}^{-1}$ body weight) (OGTT); (b) area under the time-glucose curve during the OGTT after indicated treatments for 25 weeks. Abbreviations for groups are as in Figure 1 . $n=8-11$ for each group. $P$-values are for two-way analysis of variance (ANOVA) and Student-Newman-Keuls test.
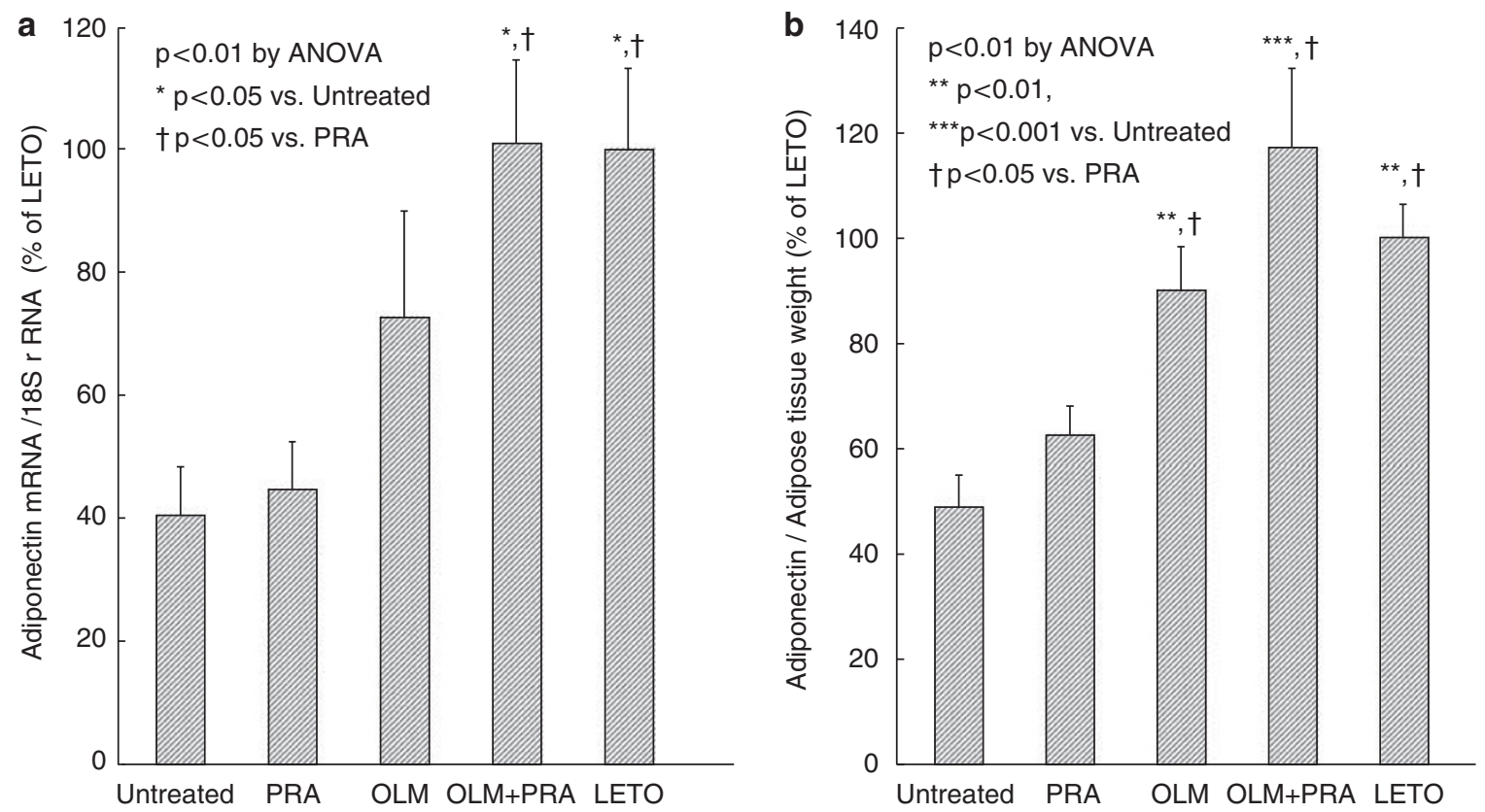

Figure 3 (a) Adiponectin messenger RNA expression in epididymal adipose tissue; (b) plasma adiponectin concentration normalized to the weight of epididymal adipose tissue after the indicated treatments for 25 weeks. Abbreviations for groups are as in Figure $1 . n=6$ for each group. $P$-values are one-way analysis of variance (ANOVA) and Student-Newman-Keuls test.

OLM, but significantly increased by OLM+PRA $(P<0.05$ vs. both the untreated controls and the PRA monotherapy group) (Figure $5 \mathrm{~b}$ ), suggesting that DHFR per eNOS content was preserved in the combined therapy group.

\section{Cardiovascular remodeling}

Figure 6a compares representative sections of intramyocardial coronary arteries at 30 weeks of age. In the untreated OLETF group, the coronary arterial wall was thickened (179\% of LETO), which was accompanied by increased perivascular fibrosis (213\% of LETO) (both $P<0.01$ vs. LETO). Olmesartan limited such structural remodeling $(P<0.05$ vs. untreated controls), which was further limited by OLM+PRA $(P<0.01$ vs. untreated controls $)$ to the level of normal LETO rats (Figure $6 \mathrm{~b}$ ).

\section{DISCUSSION}

This study showed for the first time that OLM and PRA synergistically ameliorated glucose intolerance in a rat model of metabolic syndrome, 
a

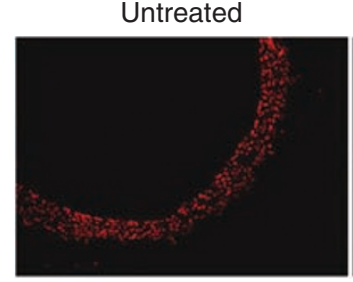

PRA

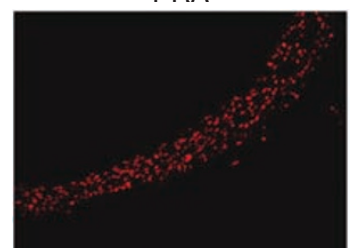

OLM

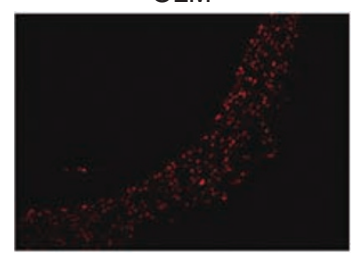

OLM+PRA

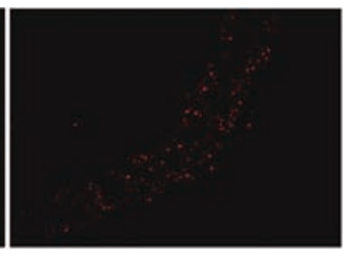

LETO

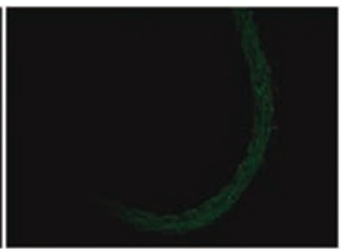

$500 \mu \mathrm{m}$

b

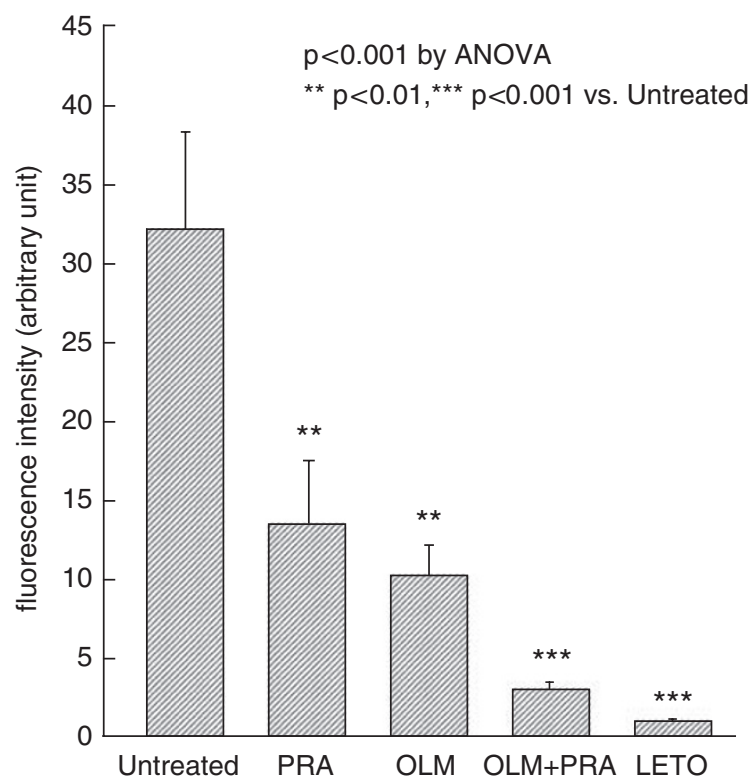

Figure 4 (a) Representative fluorescence photomicrographs of the aortic wall by dihydroethidium staining demonstrating superoxide production; (b) quantification of superoxide production based on red fluorescence intensity after indicated treatments for 25 weeks. Abbreviations for groups are as in Figure 1. To indicate the location of the specimen, green fluorescence corresponding to the elastic fibers in the aortic wall was merged with very weak red fluorescence for the Long-Evans Tokushima Otsuka (LETO). $n=4$ for each group. $P$-values are by one-way analysis of variance (ANOVA) followed by StudentNewman-Keuls test.

a eNOS

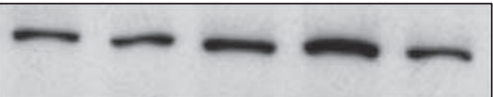
actin

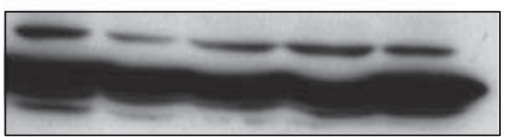
$42 \mathrm{kDa}$

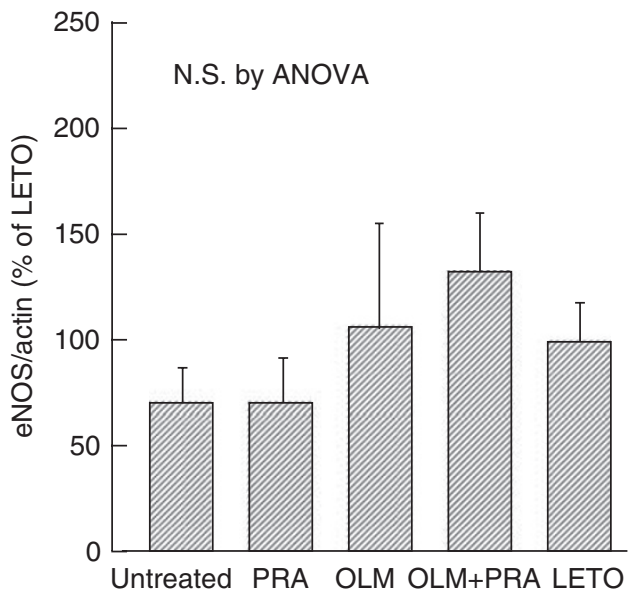

b $135 \mathrm{kDa}$

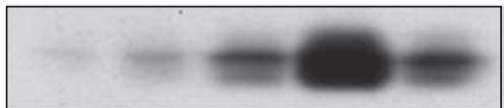
$21 \mathrm{kDa}$ actin

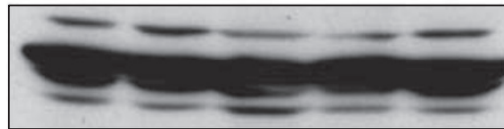
$42 \mathrm{kDa}$

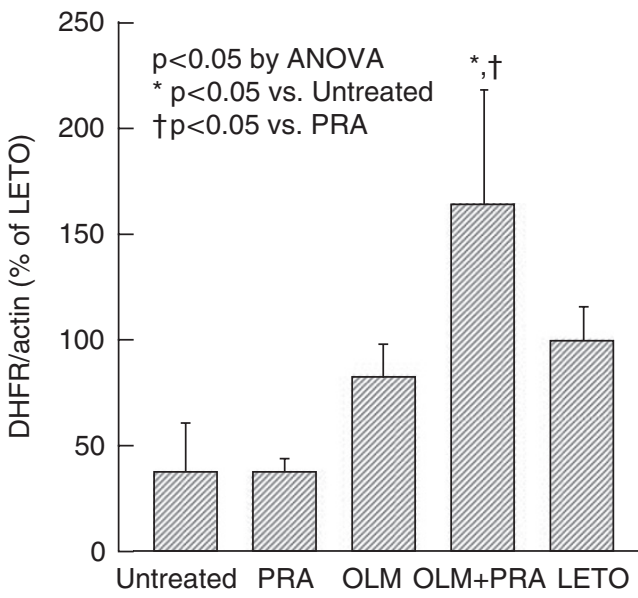

Figure 5 (a) Cardiac content of endothelial nitric oxide synthetase (eNOS); (b) cardiac content of dihydrofolate reductase determined by western blotting after the indicated treatments for 25 weeks. Abbreviations for groups are as in Figure $1 . n=5-6$ for each group for eNOS; $n=6$ for each group for dihydrofolate reductase (DHFR). P-values are for one-way analysis of variance (ANOVA) and Student-Newman-Keuls test.

which was associated with the augmentation of adiponectin messenger RNA expression in WAT and a preserved plasma adiponectin per visceral adipose tissue weight. Moreover, superoxide production in the aorta was most significantly suppressed and the cardiac content of DHFR, an eNOS coupler, was significantly increased only by the combination of the two agents. These favorable effects of the combi- 

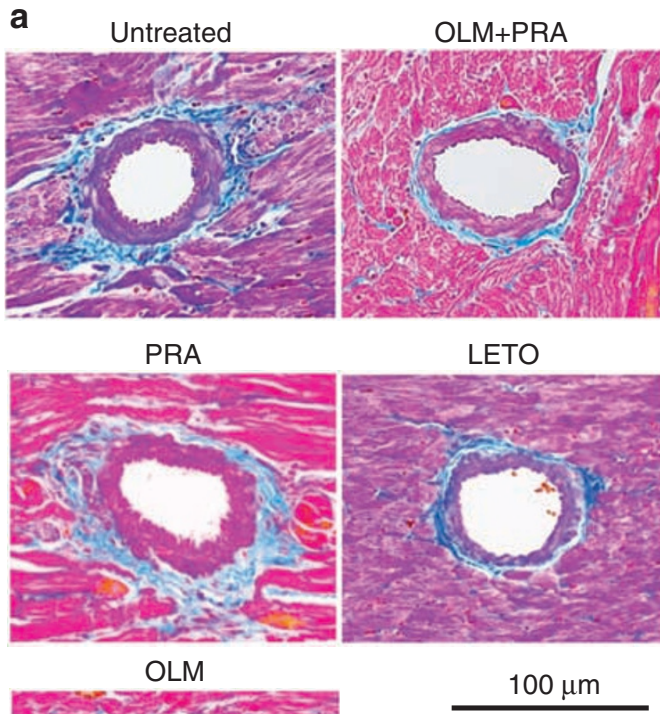
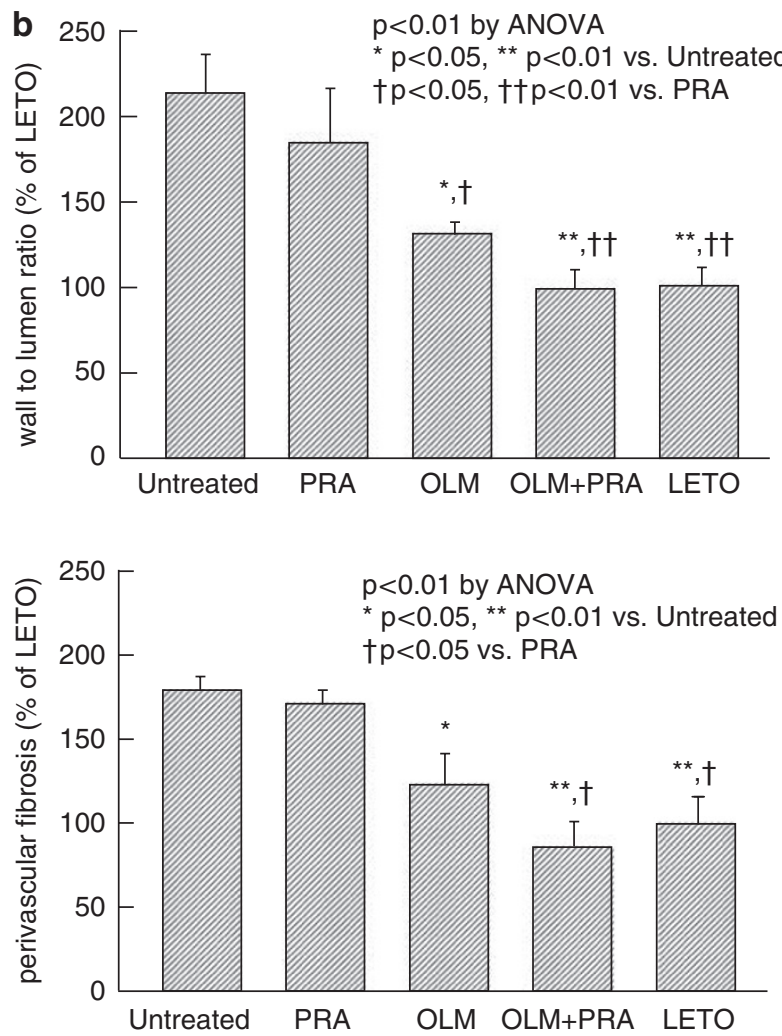

Figure 6 (a) Photomicrographs by Azan-Mallory stain comparing the extent of perivascular fibrosis and medial thickening of intramyocardial arteries; (b) results of histomorphometry using the ratio of the area of fibrosis surrounding the intramyocardial coronary arteries to the total vascular area and medial thickness as the medial wall-to-lumen ratio. Abbreviations for groups are as in Figure 1. $n=18$ specimens for each group. $P$-values are for one-way analysis of variance (ANOVA) and Student-Newman-Keuls test.

nation therapy on the metabolic and cardiovascular redox state were associated with substantial suppression of histological cardiovascular remodeling.

\section{OLETF rats as a model of metabolic syndrome}

The OLETF rat is a genetic model of hyperphagia-induced spontaneous non-insulin-dependent $\mathrm{DM}^{23}$ due to cholecystokinin-1 receptor defect. $^{24}$ This strain manifests obesity, elevated BP, insulin resistance and dyslipidemia, and thus well serves as a model of metabolic syndrome. ${ }^{10,11}$ We and others showed the consequences of such metabolic abnormalities, including impaired coronary-vasodilatory function $^{19}$ and left ventricular-diastolic dysfunction, ${ }^{17}$ which were attributable to impaired nitric oxide/superoxide balance and enhanced expression of redox-sensitive prolifero-inflammatory cytokines. ${ }^{10,11,17}$ The decreased cardiac content of DHFR and increased superoxide production in the aortic wall shown in this study adds new evidence for the accentuated oxidative stress in this model.

\section{Synergistic effect of OLM and PRA on amelioration of glucose intolerance}

New onset of DM was shown, in a clinical study, to worsen the longterm prognosis in hypertensive patients to the level of those who had been diabetic at the time of enrollment. ${ }^{25}$ In this regard, reninangiotensin system inhibitors, which prevent the development of $\mathrm{DM}$, are recommended for hypertensive patients with metabolic syndrome in several guidelines. Furthermore, because an increasing proportion of patients treated with ARB also receive statins, and because PRA is known to prevent the development of $\mathrm{DM},{ }^{5}$ the synergistic effects of an ARB and PRA on preventing progressive glucose intolerance warrant investigation. Here, we performed a preclinical study, in which the OLETF rats were treated with OLM and/or PRA over the long term. After a 25-week treatment, reduction of the area under the time-glucose curve during OGTT reached a significant level only by the combination of the doses, which did not achieve statistically significant amelioration as monotherapy. Thus, the two agents synergistically prevented the progression of glucose intolerance in the metabolic syndrome model.

Among several possible mechanisms involved in the improved glucose tolerance, we examined the expression of adiponectin, an adipose-derived secretory factor that exerts insulin-sensitizing effects. We found that adiponectin messenger RNA levels were significantly augmented to the normal level only when the two agents were combined, whereas OLM monotherapy showed only sub-significant effect. Similarly, the combination therapy more significantly increased plasma adiponectin/adipose tissue weight ratio than the monotherapy with OLM.

Whereas oxidative stress induced by angiotensin II infusion reduced the plasma adiponectin concentration, ${ }^{26}$ OLM was shown to preserve adiponectin messenger RNA expression in the WAT of obesity models at least by anti-oxidative actions. ${ }^{4}$ Therefore, the OLM-enhanced adiponectin expression observed in our study was presumably achieved by attenuating superoxide production through blockage of AT1-mediated NADPH oxidase activation in macrophages and endothelial cells present in hypertrophic WAT, as shown earlier. ${ }^{4}$ 
OLM was also reported to ameliorate impaired adiponectin secretion from isolated adipocytes exposed to hydrogen peroxide, suggesting its direct adipotropic action. ${ }^{4}$

On the other hand, it is well established that statins exert antioxidative effects against angiotensin II-stimulated superoxide production by inhibiting isoprenylation of low-molecular-weight G-proteins, such as Racl, which is essential for NADPH oxidase activation. Moreover, PRA also has been shown to upregulate adiponectin messenger RNA expression in 3T3-L1 adipocytes, ${ }^{9}$ although the mechanism remains unknown.

Thus, it is speculated that the inhibition of NADPH oxidase activation in WAT by both agents and agent-specific direct actions on adipocytes might contribute to the synergistic augmentation of adiponectin expression, which played a role in the improvement of glucose tolerance in this study.

\section{Amelioration of cardiovascular redox state}

The cardiac eNOS content was not decreased in the OLETF rats, as shown before, ${ }^{11}$ and not up-regulated by any treatment including PRA monotherapy, which had increased eNOS content at a higher dose $\left(100 \mathrm{mg} \mathrm{kg}^{-1}\right.$ per day) in our earlier study. ${ }^{11}$ The eNOS can also produce superoxide when it is 'uncoupled'. Therefore, the eNOS content does not necessarily parallel NO production, which is dependent on the magnitude of eNOS uncoupling. One of the mechanisms involved in eNOS uncoupling is a deficiency of BH4, which is essential for eNOS dimerization. In $\mathrm{BH} 4$ deficiency, electron flow from eNOS flavins becomes uncoupled from L-arginine oxidation, resulting in the formation of superoxide by the oxygenase domain. ${ }^{14}$ In DM, oxidation of $\mathrm{BH} 4$ to $\mathrm{BH} 2$ is enhanced, and DHFR, which preserves $\mathrm{BH} 4$ through reduction of $\mathrm{BH} 2$ to $\mathrm{BH} 4$, is downregulated, thereby impairing the endothelial function. ${ }^{16}$ In the OLETF rats, the DHFR content, used as a marker of eNOS-recoupling, was decreased to $40 \%$ of normal control, whereas the total eNOS content did not differ from the control, suggesting that eNOS is readily uncoupled in this model.

OLM and PRA were reported to exert anti-oxidative effects and upregulate DHFR independently in a salt-sensitive hypertension model, ${ }^{13}$ in which OLM prevented the downregulation of DHFR more than PRA, whereas Akt phosphorylation was enhanced by PRA but not OLM, indicating differential pleiotropic effects between the two agents. ${ }^{13}$

In the model of metabolic syndrome we found that cardiac DHFR decrease was significantly prevented only on achieving significant improvement of glucose tolerance. Improved hyperglycemia, presumably, further suppressed oxidative stress, which might augment subsignificant DHFR upregulation by each agent to the significant level, attaining eNOS recoupling in the present study. Thus, the more amelioration of redox state elicited by PRA+OLM would have suppressed redox-sensitive gene expression, which yielded the more significant attenuation of remodeling of intramyocardial coronary arteries than monotherapy.

Although PRA monotherapy modestly improved hyperglycemia and decreased superoxide production as assessed with the sensitive DHE staining, it failed to upregulate cardiac DHFR and to prevent histological remodeling. These modest and partial effects of PRA monotherapy at this dose (30\% of the full dose, which had shown eNOS upregulation in our earlier study), ${ }^{11}$ seemed not to be sufficient to improve $\mathrm{NO} /$ superoxide balance leading to histological remodeling. Nevertheless, non-linear augmentation of these parameters by the combination therapy may suggest unknown mechanisms for the synergistic effects, including possible alteration in bioavailability of the respective agent and their cross-talks in the intracellular signaling pathways.

\section{Lipid-lowering and antihypertensive effects}

The tested doses of PRA and OLM exhibited significant lipid-lowering and antihypertensive effects, respectively, which successfully reproduced their clinical achievements in treating patients with metabolic syndrome. Interestingly, OLM exerted even greater lipid-lowering effect than PRA, which enhanced the effect of PRA in combination. Angiotensin II infusion induces hypercholesterolemia in fructose-fed rats, which is rectified by OLM, ${ }^{27}$ whereas in the OLETF rats, angiotensinogen and AT1 expressions in the liver are both increased. ${ }^{28}$ These reports imply substantial contribution of the activated AT1 pathway to hypercholesterolemia in rat obese models. At least in the OLETF rats, inhibition of this AT1-mediated mechanism by OLM at $3 \mathrm{mg} \mathrm{kg}^{-1}$ per day was greater than the modest HMG-CoA reductase inhibition by PRA at $30 \mathrm{mg} \mathrm{kg}^{-1}$ per day.

Another notable finding was that PRA significantly enhanced the antihypertensive effect of OLM. This might be partly attributable to the limitation of eNOS uncoupling by the combination therapy. This also agrees with the finding that statin reversed the increased expression of AT1 and the augmented BP response to angiotensin II in hypercholesterolemic men. ${ }^{29}$ Thus, it is speculated that both hypercholesterolemia and hypertension would be closely linked to augmented AT1 stimulation in this model. Although further mechanisms remain unknown, our findings imply that the combination of OLM and PRA will clinically benefit lipid and BP control per se in patients with metabolic syndrome.

\section{Study limitations}

Although we attributed the amelioration of glucose tolerance to improved insulin sensitivity because of adiponectin upregulation, direct activation of the insulin-signaling pathway by either drug could have contributed. In addition, because further improvement of the insulin-resistance index over monotherapy by the combination therapy was not found, improved pancreatic $\beta$-cell function might also have contributed to the improved glucose tolerance in this study.

Precisely how the combination of OLM and PRA upregulated adiponectin messenger RNA expression in WAT remains unknown. As WAT weight was decreased by the combined therapy, it is speculated that adipocyte hypertrophy was suppressed along with adiponectin upregulation in this group. Precise cell-size assessments would have provided adipocyte-size distribution, which also remains to be clarified. Furthermore, studies using adipocytes in vitro or cocultures of adipocytes with macrophages or endothelial cells to investigate the effects of OLM and PRA would provide further information on direct adipotropic or inter-cellular mechanisms. The plasma level of high-molecular-weight adiponectin rather than total adiponectin would have provided a more accurate assessment.

We only measured total eNOS content, but did not assess its dimerization or phosphorylation, which would have better represented eNOS activity. Similarly, because DHFR content was shown to parallel eNOS dimerization in the similar treatments, ${ }^{13}$ we also did not assess $\mathrm{BH} 4$ content per se, which would have yielded robust evidence of improved cardiovascular redox state based on eNOS recoupling by the combination therapy.

Lastly, we only tested a combination of sub-maximal doses of OLM and PRA. In our earlier studies, a high dose $\left(100 \mathrm{mg} \mathrm{kg}^{-1}\right.$ per day $)$ of PRA almost completely prevented new-onset DM in this model, ${ }^{10,11}$ and this was accompanied by augmented adiponectin messenger RNA expression in $\mathrm{WAT}^{11}$ and an increased cardiac eNOS content. ${ }^{10,11}$ Therefore, combining higher doses, aside from the possible adverse effects, would probably not be synergistic or additive. Thus, further studies are warranted to extrapolate the present findings to combina- 
tions of other doses of OLM and PRA or of other ARBs and statins, not only in animal models but also in patients.

\section{Conclusions}

Olmesartan plus pravastatin synergistically prevented progressive glucose intolerance in a rat model of metabolic syndrome. This effect was associated with enhanced adiponectin expression in WAT, an ameliorated cardiovascular redox state and resultant diabetic remodeling. Thus, the present findings justify the administration of combined ARBs and statins in treating patients with multiple risk factors including hypertension, dyslipidemia and glucose intolerance, or with metabolic syndrome.

\section{ACKNOWLEDGEMENTS}

This work was partly supported by a research grant from Daiichi-Sankyo Co. Ltd., Tokyo, Japan. The authors are grateful to Otsuka Pharmaceutical Co. Ltd., Tokyo, Japan for providing the OLETF rats and LETO rats at no cost.

1 Yusuf S, Gerstein H, Hoogwerf B, Pogue J, Bosch J, Wolffenbuttel BH, HOPE Study Investigators. Ramipril and the development of diabetes. JAMA 2001; 286: 18821885.

2 Scheen AJ. Renin-angiotensin system inhibition prevents type 2 diabetes mellitus. Part 1. A meta-analysis of randomised clinical trials. Diabetes Metab 2004; 30: 487-496.

3 Nishimura H, Sanaka T, Tanihata Y, Naito T, Higuchi C, Otsuka K. Losartan elevates the serum high-molecular weight-adiponectin isoform and concurrently improves insulin sensitivity in patients with impaired glucose metabolism. Hypertens Res 2008; 31: 1611-1618

4 Kurata A, Nishizawa H, Kihara S, Maeda N, Sonoda M, Okada T, Ohashi K, Hibuse T, Fujita K, Yasui A, Hiuge A, Kumada M, Kuriyama H, Shimomura I, Funahashi T. Blockade of angiotensin II type-1 receptor reduces oxidative stress in adipose tissue and ameliorates adipocytokine dysregulation. Kidney Int 2006; 70: 1717-1724.

5 Freeman DJ, Norrie J, Sattar N, Neely RD, Cobbe SM, Ford I, Isles C, Lorimer AR, Macfarlane PW, McKillop JH, Packard CJ, Shepherd J, Gaw A. Pravastatin and the development of diabetes mellitus: evidence for a protective treatment effect in the West of Scotland Coronary Prevention Study. Circulation 2001; 103: 357-362.

6 Ishikawa M, Namiki A, Kubota T, Yajima S, Fukazawa M, Moroi M, Sugi K. Effect of pravastatin and atorvastatin on glucose metabolism in nondiabetic patients with hypercholesterolemia. Intern Med 2006; 45: 51-55.

7 Mita $T$, Watada $H$, Nakayama $S$, Abe $M$, Ogihara $T$, Shimizu $T$, Uchino $H$, Hirose $T$, Kawamori R. Preferable effect of pravastatin compared to atorvastatin on beta cell function in Japanese early-state type 2 diabetes with hypercholesterolemia. Endocr $\mathrm{J}$ 2007; 54: 441-447.

8 Sugiyama S, Fukushima H, Kugiyama K, Maruyoshi H, Kojima S, Funahashi T, Sakamoto T, Horibata Y, Watanabe K, Koga H, Sugamura K, Otsuka F, Shimomura I, Ogawa H. Pravastatin improved glucose metabolism associated with increasing plasma adiponectin in patients with impaired glucose tolerance and coronary artery disease. Atherosclerosis 2007; 194: e43-e51.

9 Takagi T, Matsuda M, Abe M, Kobayashi H, Fukuhara A, Komuro R, Kihara S, Caslake MJ, McMahon A, Shepherd J, Funahashi T, Shimomura I. Effect of pravastatin on the development of diabetes and adiponectin production. Atherosclerosis 2008; 196: 114-121.

10 Chen Y, Ohmori K, Mizukawa M, Yoshida J, Zeng Y, Zhang L, Shinomiya K, Kosaka H, Kohno M. Differential impact of atorvastatin vs pravastatin on progressive insulin resistance and left ventricular diastolic dysfunction in a rat model of type II diabetes. Circ J 2007; 71: 144-152.

$11 \mathrm{Yu}$ Y, Ohmori K, Chen Y, Sato C, Kiyomoto H, Shinomiya K, Takeuchi H, Mizushige $\mathrm{K}$, Kohno M. Effects of pravastatin on progression of glucose intolerance and cardiovascular remodeling in a type II diabetes model. J Am Coll Cardiol 2004; 44: 904-913.

12 Li Z, Iwai M, Wu L, Liu HW, Chen R, Jinno T, Suzuki J, Tsuda M, Gao XY, Okumura M, Cui TX, Horiuchi M. Fluvastatin enhances the inhibitory effects of a selective AT1 receptor blocker, valsartan, on atherosclerosis. Hypertension 2004; 44: 758-763.

13 Yamamoto E, Yamashita T, Tanaka T, Kataoka K, Tokutomi Y, Lai ZF, Dong YF, Matsuba $\mathrm{S}$, Ogawa H, Kim-Mitsuyama S. Pravastatin enhances beneficial effects of olmesartan on vascular injury of salt-sensitive hypertensive rats, via pleiotropic effects. Arterioscler Thromb Vasc Biol 2007; 27: 556-563.

14 Alp NJ, Channon KM. Regulation of endothelial nitric oxide synthase by tetrahydrobiopterin in vascular disease. Arterioscler Thromb Vasc Biol 2004; 24: 413-420.

15 Chalupsky K, Cai H. Endothelial dihydrofolate reductase: critical for nitric oxide bioavailability and role in angiotensin II uncoupling of endothelial nitric oxide synthase. Proc Natl Acad Sci USA 2005; 102: 9056-9061.

16 Oak JH, Cai H. Attenuation of angiotensin II signaling recouples eNOS and inhibits nonendothelial NOX activity in diabetic mice. Diabetes 2007; 56: 118-126.

17 Mizushige K, Yao L, Noma T, Kiyomoto H, Yu Y, Hosomi N, Ohmori K, Matsuo H. Alteration in left ventricular diastolic filling and accumulation of myocardial collagen at insulin-resistant prediabetic stage of a type II diabetic rat model. Circulation 2000; 101: 899-907.

18 Guzik TJ, Mussa S, Gastaldi D, Sadowski J, Ratnatunga C, Pillai R, Channon KM. Mechanisms of increased vascular superoxide production in human diabetes mellitus: role of NAD(P)H oxidase and endothelial nitric oxide synthase. Circulation 2002; 105 : 1656-1662.

19 Yu Y, Ohmori K, Kondo I, Yao L, Noma T, Tsuji T, Mizushige K, Kohno M. Correlation of functional and structural alterations of the coronary arterioles during development of type II diabetes mellitus in rats. Cardiovasc Res 2002; 56: 303-311.

20 Hoffested J, Arvidsson E, Sjolin E, Wahlen K, Arner P. Adipose tissue adiponectin production and adiponectin serum concentration in human obesity and insulin resistance. J Clin Endocrinol Metab 2004; 89: 1391-1396.

21 Ribot J, Rodríguez AM, Rodríguez E, Palou A. Adiponectin and resistin response in the onset of obesity in male and female rats. Obesity (Silver Spring) 2008; 16: 723-730.

22 Bullen Jr JW, Bluher S, Kelesidis T, Mantzoros CS. Regulation of adiponectin and its receptors in response to development of diet-induced obesity in mice. Am J Physiol Endocrinol Metab 2007; 292: E1079-E1786.

23 Kawano K, Hirashima T, Mori S, Saitoh Y, Kurosumi M, Natori T. Spontaneous longterm hyperglycemic rat with diabetic complications. Otsuka Long-Evans Tokushima Fatty (OLETF) strain. Diabetes 1992; 41: 1422-1428.

24 Takiguchi S, Takata Y, Funakoshi A, Miyasaka K, Kataoka K, Fujimura Y, Goto T, Kono A. Disrupted cholecystokinin type-A receptor (CCKAR) gene in OLETF rats. Gene 1997; 197: 169-175.

25 Verdecchia P, Reboldi G, Angeli F, Borgioni C, Gattobigio R, Filippucci L, Norgiolini S, Bracco C, Porcellati C. Adverse prognostic significance of new diabetes in treated hypertensive subjects. Hypertension 2004; 43: 963-969.

26 Ran J, Hirano T, Fukui T, Saito K, Kageyama H, Okada K, Adachi M. Angiotensin II infusion decreases plasma adiponectin level via its type 1 receptor in rats: an implication for hypertension-related insulin resistance. Metabolism 2006; 55 : 478-488.

27 Hirano T, Ran J, Adachi M. Opposing actions of angiotensin II type 1 and 2 receptors on plasma cholesterol levels in rats. J Hypertens 2006; 24: 37-38.

28 Kurita S, Takamura T, Ota T, Matsuzawa-Nagata N, Kita Y, Uno M, Nabemoto S, Ishilura $\mathrm{K}$, Misu H, Ando H, Zen Y, Nakamura Y, Kaneko S. Olmesartan ameliorates a dietary rat model of non-alcoholic steatohepatitis through its pleiotropic effects. Eur J Pharm 2008; 588: 316-324.

29 Nickenig G, Bäumer AT, Temur Y, Kebben D, Jockenhövel F, Böhm M. Statin-sensitive dysregulated AT1 receptor function and density in hypercholesterolemic men. Circulation 1999; 100: 2131-2134. 